\title{
Idade Cronológica: mera questão referencial no processo de envelhecimento
}

\author{
Lúcia Regina Severo Duarte
}

\begin{abstract}
Resumo
O presente trabalho propõe uma breve revisão bibliográfica sobre alguns aspectos da velhice, tendo em vista que hoje em dia o tema terceira idadesenectude está se convertendo numa realidade social com a qual a cada dia mais vamos conviver. Destacamos alguns aspectos que consideramos relevantes como: evolução histórica da velhice, os mitos, a saúde/enfermidade e em especial a questão IDADE, não somente idade cronológica, do ponto de vista referencial, mas sim as idades dentro de um proceso dinâmico; idade social, biológica e psicológica.

Palavras-Chave: mitos; idade cronológica; idade social; idade biológica; idade psicológica.
\end{abstract}

\begin{abstract}
In this research we propose a bibliographic review of the diferent aspects that make reference to old age. This subject is considered very important because it is a social reality that sometimes is relegated to a lower level. During the research we face a sort historicak evolution of aging with its myths and stereotypes. If we talk about aging its worth making reference to the concepts of health/illness. We will find as a first question the ideas about "health" and "illnes" like opposite phenomena. However, the problem is not that simple. Later on, we will arrive to our principal objetive in wich we will make reference to old age as a differential process and not as a life stage. It is impossible that the majority of the people would generalize but, we will be always in frot of differential and social process that has permittes to enhance a sort of characteristics that make easy its comprehencion; where the given value to the chronological age is just
\end{abstract}

\footnotetext{
${ }^{1}$ Psicóloga - Professora da Pontifícia Universidade Católica do Rio Grande do Sul, Porto Alegre, RS Doutoranda de Psicología Clínica y de la Salud. Universidad Autónoma de Madrid, España.

E-mail: 1severo@sinix.net
}

Estud. interdiscip. envelhec., Porto Alegre, v.2, p.35-47, 1999. 
use as a regular point of reference. We will continue with a study of ages: the social age, the biological age and the psychological age, each on with own advantages and its own limits; they are still complementary being the origin ofs dynamic process.

Key-Words: myths; chronological age; social age; biological age; psychological age.

\section{Introdução}

Historicamente, para a maior parte da população, a velhice é sinônimo de pobreza no aspecto econômico, inatividade no laboral, marginalidade na vida social e cultural, enfermidade no âmbito da saúde. A partir da segunda guerra mundial, observa-se uma enorme modificação na situação existente até então. Hoje, pessoas com idade mais avançada participam cada vez mais ativamente na vida social, de atividades culturais, de lazer e físicas, vivem mais anos e com melhores condições de saúde. Com o aumento gradual de esperança de vida e a diminuição da taxa de natalidade em muitos países, os problemas de saúde da população, a longo prazo, podem transbordar, inclusive, com previsões mais pessimistas, levando a um desmoronamento dos sistemas de assistência sanitária e social.

Desde uma perspectiva estoicista, se argumentou positivamente sobre a velhice no Diálogo Sobre a Velhice de Kio e no Diálogo da Velhice, de Cicero. Especialmente neste último, onde argumenta, de maneira definitiva, a favor da velhice. Se concedia para a idade avançada, idoneidade naquelas funções que haviam desempenhado durante a vida. Aparece, também, um resumo do que se considerava próprio do envelhecimento fisiológico, com presença de conceitos e conselhos sobre atividade física, sexual, social e até política, do ancião, que podiam muito bem ter vigência hoje em dia. Não existe um consenso e de quando foi o início do estudo científico sobre o envelhecimento. No entanto, Birren (1960) situa este início entre 1835 e 1918, Comfort (1979) entre 1561 e 1626, pela publicação do livro de Bacon, History of Life and Death.

Existem numerosas idéias sobre o envelhecimento que formam parte de 
nossa cultura. A filosofia, as artes e a literatura de alguma maneira recorrem a esta bagagem. Dentro delas, uma linha de pensamento reflete a crença de que prolongar a vida não é possível, nem desejável, outra linha alternativa enfatiza o desejo de prolongar a vida. São duas formas de pensar que se encontram enraizadas com as teorias passadas e atuais sobre o envelhecimento (Kart, 1990; Rodríguez, 1989), duas formas opostas de pensar que recorrem a realidade e o desejo, que formam parte de numerosos mitos e idéias culturais sobre o envelhecimento.

A idéia central sobre o envelhecimento na atualidade percebe que o importante não é a longevidade (a imortalidade), mas sim a qualidade de vida. Uma das frases criadas recentemente pela Organizaçao Mundial de Saúde (OMS) e que enfatiza esta perspectiva afirma que "o importante não é dar anos à vida, mas sim vida aos anos", ou seja, o crucial não é a longevidade, mas sim manter a qualidade de vida. A permanência destas idéias nos sugere a dificuldade que resulta, as vezes, distinguir entre a realidade e a história, entre o mito e a ciência.

O envelhecimento é um fenômeno universal que teve lugar em todas as épocas, culturas e civilizações. $\mathrm{O}$ conhecimento que temos atualmente a respeito é o resultado da acumulação de experiência cultural e científica ao longo da história. A velhice é uma etapa que podiamos dizer está relacionada com a idade adulta; seu conhecimento científico todavia forma parte de uma ciência "jovem" (Vega; Bueno, 1996).

Mas, apesar do envelhecimento ser um fenômeno universal, são poucos os conhecimentos sobre este processo, existindo por parte de muitos uma visão estereotipada. Para muitos os velhos são considerados enfermos, incapazes, improdutivos, dependentes, rígidos, dogmáticos, desmemoriados e teimosos. Afortunadamente, estamos hoje muito longe desta concepção da "velhice como desastre" e de identificar o processo de envelhecimento com a demência senil. Como ocorre em todas as etapas de desenvolvimento psicológico, a que agora ocupa-nos é tão complexa como pluridimensional. O envelhecimento é um fato biológico, social e psicológico. Qualquer consideração que não se acente simultaneamente sobre estes pilares, corre o risco de deformação e de dar lugar a crenças condenadas a uma rápida substituição. Para Carretero; Palacios; 
Marchesi (1995), o envelhecimento é inquestionável, é um fato biológico previsto em nosso código genético; forma parte de um plano madurativo próprio da espécie com tanta invariabilidade como a substituição dos dentes de leite pelos definitivos ou como as modificações biológicas na puberdade transformam o corpo infantil em adulto. $\mathrm{O}$ envelhecimento e a morte são tão circunstanciais ao ser humano como o crescimento.

\section{Saúde e Enfermidade}

Se falamos de velhice cabe fazer referência aos conceitos de saúde/ enfermidade. Tendo como primeira questão as idéias de "saúde" e "enfermidade" como fenômenos contrapostos. No entanto, o problema não é assim tão simples; a saúde não deve ser entendida unicamente como ausência de enfermidade, mas sim como algo positivo em si mesmo (a saúde se entende como um estado de bem estar físico e social) que deve promocionar-se mediante condutas apropriadas (ver Belloch,1989). De fato, esta idéia central foi em grande parte a responsável de que emergisse na ciência psicológica (basicamente a do tipo aplicado) a área denominada "Psicologia da Saúde".

$\mathrm{O}$ conceito de enfermidade vem sendo dado pela diferenciação entre o subjetivo e o objetivo, em outros termos, entre os sintomas e o que representam, respectivamente. $\mathrm{O}$ estado de enfermo é um fenômeno altamente social e pessoal. A diferença entre os conceitos subjetivos e objetivos de enfermidade está claramente estabelecida na língua anglosaxônica mediante os termos illness e disease. O primeiro usualmente se emprega para referir que uma determinada pessoa sente-se mal (sente-se enferma) e leva a cabo atividades que refletem o problema, tais como, ficar na cama ou tomar medicamentos. Diante destas circunstâncias, o indivíduo tende a adotar o "papel de enfermo". O termo disease, em contraste, se utiliza para denominar um estado objetivo de transtorno orgânico, por exemplo, presença de isquemia miocárdica. Desta forma, um indivíduo que mostre condutas de enfermidade, que sente-se enfermo (illness), pode possuir ou não uma enfermidade orgânica (disease) e uma pessoa que possua problemas orgânicos objetivos pode ou não exibir uma conduta de enfermidade.

Vários autores vêm se preocupando com o conceito de saúde, saúde/ 
enfermidade. Para muitos saúde é não ter uma enfermidade, para outros, por exemplo, para Orem (1980), um indivíduo pode se encontrar bem em um estado sadio ou enfermo, sendo as alternativas mutuamente exclusivas. Outro grupo de autores definem a saúde como algo contínuo (dinâmico), o que implicaria considerá-la como um aspecto final de uma alteração progressiva; ou seja, a ausência de patologia constitui um extremo e estar são o outro. Um autor partidário desta orientação é San Martín (1983), para quem passar de um estado de saúde ao da enfermidade e vice-versa se produz de uma forma gradual e contínua.

Um terceiro grupo considera a saúde como algo utópico; quer dizer, como um estado de bem estar e autorrealização que é praticamente impossível de alcançar. Dentro deste aspecto se inclui a definição da Organização Mundial de Saúde, que conceitualiza a saúde como "( . . . ) um estado completo de bem estar físico, mental e social, e não meramente a ausência de invalidez ou enfermidade." (Hogarth, 1978). Muitos outros autores consideram que o parâmetro mais relevante da saúde é a "adaptação". Sendo assim, um indivíduo, ao menos em princípio, se encontra adaptado ao meio se desempenha de forma adequada o papel que lhe corresponde. Isto leva a considerar a saúde desde uma perspectiva das funções e a defini-la de acordo como o indivíduo desempenha suas funções sociais (Ahmed; Kolker; Coelho, 1979; Parsons, 1976).

Jahoda (1958), Maslow (1979), Taylor (1991), Warr (1987), desde uma perspectiva mais sociológica, centrando-se de uma forma prioritária na saúde mental positiva e tendo em conta diferentes esferas do funcionamento do indivíduo, incluem no conceito de saúde as seguintes características: a) êxito ou aspiração; b)funções cognitivas; c) equilíbrio emocional; d) ajuste social; e) autoestima e f) ilusões.

Antonowisky (1987) expõe o Modelo Salutogênico, dizendo que não é antagônico, mas sim complementário do modelo patológico, apresenta as seguintes características: a) considera saúde/enfermidade como um contínuo; b) dá ênfases a história saúde/enfermidade do ser humano; c) valoriza os recursos de enfrentamento para a superação dos problemas e promoção da saúde e d) fomenta a adaptação crítica de organismo-ambiente. A Organização Mundial 
de Saúde (1986); dentro de um marco de "saúde para todos no ano 2000", também sugere a perspectiva de centrar-se nos componentes positivos da saúde.

\section{Terceira Idade-Senectude}

Aragó (1995) faz referência a aspectos importantes quando diz que a terceira idade-senectude deve ser vista como um processo diferencial e não como um estado. Dizer que é um processo equivale a afirmar que não é algo que acontece de repente, como poderia ser um acidente ou uma enfermidade que surge subitamente. Estamos entre uma modificação gradual e universal. É possível fazer generalizações, mas sempre estaremos ante um processo diferencial. Por exemplo, aos 70 anos algumas pessoas conservam seu vigor físico, sua capacidade intelectual, sua curiosidade científica, etc., e outras menos. Dependerá, entre outras causas, do ambiente onde viveram, se foi mais ou menos estimulante, do tipo de profissão exercida, do êxito ou fracasso em suas tarefas, etc.

Entre as variáveis diferenciadoras que atuam de uma forma mais geral e conhecida, cabe assinalar o chamado efeito cohorte ou efeito de gerações: quer dizer, o conjunto de circunstâncias histórico-culturais que marcam o nascimento e educação de cada um. Também, como fatores gerais que de algum modo diferenciam os grupos de indivíduos, cabe assinalar o que coloca Erikson (1959) com sua teoria das oito idades do homem, ou seja, o modo como cada um realizou suas próprias tarefas nos diversos períodos evolutivos. Refere, ainda, que em cada época ou idade da vida ocorrem acontecimentos próprios com sua vertente positiva e alguns riscos ou possíveis negatividades, mas insiste em que uma idade determinada não é mero resíduo do passado, senão que tem sua própria dinâmica; é óbvio que o passado condiciona e as vezes fortemente, mesmo assim isto não é impedimento para que se atue em cada período com virtualidade e possibilidades novas.

Que a senectude seja um processo diferencial indica também que não só as realidades objetivas, que com sua presença e ação vão marcando e modelando a existência de cada indivíduo, mas sim de forma muito especial o modo como se captam e vivenciam as expectativas que se vão formando, a maneira como se avaliam os próprios acontecimentos e os alheios, é o que de fato molda, 
configura e diferencia a terceira idade e senectude.

É importante tomar em consideração os fatores sociais dentro de um processo diferencial de envelhecimento, estes fatores são tanto do tipo micro como macro social que favorecem ou dificultam durante o curso da vida; são fatores principalmente sociais que contribuem poderosamente para modelar a biografia de cada pessoa e cujos resultados cumulativos ou compensatórios vão se apreciando no transcurso dos anos e imprimem um rumo típico da velhice. Lehr (1980) adverte que o próprio indivíduo tem parte da responsabilidade de sua situação. Estamos, pois, ante uma causalidade complexa que esta mesma autora qualifica de " ( . . . ) auto causalidade determinada pela sociedade."

A noção de terceira idade e senectude como um processo diferencial e social tem permitido que surjam uma série de características que facilitam sua compreensão e abrem um leque de possibilidades mais otimistas. $\mathrm{O}$ valor atribuído à idade cronológica só "representa um índice global e sintético indiferenciado: é aglutinante cômodo para designar um conjunto de fatores em complexa interação que atuam ao longo de um transcurso temporal" (Coll, 1979; Wohlwill, 1973). A variável idade é um indicador do que ocorre somente num período cronológico determinado; é necessário levar em conta que, segundo a conduta que se trate, ou seja, os limites mínimo e máximo entre as modificações comportamentais, podem estar muito distanciados. Isto significa que a idade somente tem uma função referencial. Segundo Trautner (1979), a idade como tal não é uma magnitude psicológica.

Não se pode atribuir somente à idade qualquer tipo de deterioro e, menos ainda será legítimo homogeneizar todo o processo de envelhecimento, unificálo como um todo; todavia, menos sensato será reduzi-lo ou equipará-lo a uma parte do mesmo, especialmente se esta parcela é claramente patológica como seria o caso da senilidade entendida como demência senil. Ainda que isto pareça óbvio, é nesta redução ou equiparação que se vem apoiando muitos estereótipos populares; englobam como conceito de decreptude todo o processo de envelhecimento, dificultando e impedindo uma correta compreensão da evolução humana.

Sendo a velhice hoje em dia bastante prolongada, chegando algumas 
vezes a ocupar até um terço da vida, parece conveniente assinalar uns períodos que marcam este processo, referindo-se estas divisões a uma situação concreta. Estas etapas podiam ser: a) terceira idade: em torno dos 65 anos aproximadamente; anos que precedem a aposentadoria; b) ancianidade: pode centrar-se entre os 70-75 anos, momento em que ocorrem modificações no sentido de orientação de algumas tarefas e reponsabilidades. Período muitas vezes com presença de limitações físicas e sociais; c) última senectude: situada ao redor dos 80 anos. Pode haver possibilidade de viver este período com grande plenitude; no entanto, é um período caracterizado, particularmente, por limitações de todo o tipo e a proximidade da morte.

No parágrafo anterior, viu-se a divisão do ponto de vista cronológico, no entanto, sabe-se que a idade cronológica serve de ponto referencial habitual, tipifica as pessoas baseando-se na mera idade cronológica. Birren (1959), preferiu falar de idades: idade social, idade biológica e idade psicológica. À continuação cada uma delas será trabalhada.

\subsection{Idade Social}

Para Aragó (1995), a idade social considera especialmente o indivíduo como membro do grupo-grupos dos quais pertence e da sociedade que lhes acolhe; atende também as variações das interrelações. Por isto, a idade social costuma medir-se pela capacidade funcional em contribuir no trabalho, a proteção do grupo ou grupos que pertence e a sociedade que os acolhe; atende a dívida que pode reportar o indivíduo frente ao grupo social ao qual pertence. A estimativa desta utilidade depende em grande parte das medidas legais, valores em uso e também dos prejuízos e estereótipos dominantes. Neugarten; Datan (1973) fazem referência a esta idéia expressando:

“( . . ) os graus de idade são expressões de fato de que todas as sociedades racionalizam os passos do tempo da vida, o dividem em unidades socialmente relevantes e assim se pode dizer que transformam o tempo do calendário (o tempo biológico) em tempo social... Os sistemas de critérios e situações legais baseados nos anos, que emergem em todas as sociedades, deveres, direitos e 
recompensas se distribuem diferencialmente aos grupos de idade que eles mesmos haviam definido socialmente." (p.294).

De acordo com o que nos coloca Mishara; Riedel (1995), a idade social designa papéis que se pode, se deve, se pretende e se deseja que venham a desempenhar na sociedade. Determinados papéis sociais podem entrar em conflito com aspectos arbritrários da idade cronológica. O conflito entre as idades social, psicológica e cronológica constitui uma forma de dissonância. Certas variáveis sociais evoluem com a idade, mas sem seguir necessariamente a idade cronológica. A variável indepência/dependência é um exemplo deste aspecto.

\subsection{Idade Biológica}

A idade biológica, ou seja, as modificações físicas e biológicas podem servir para definir o envelhecimento. Mas, a menos que se produza um traumatismo excepcional, o envelhecimento físico como refere Mishara; Riedel (1995), “( . . . ) desenvolve-se gradualmente, acrescentando que resulta ser arbitrário precisar o momento em que uma pessoa é fisicamente velha." (p.24). O envelhecimento evoca, habitualmente, mudanças físicas desagradáveis: perda de força, diminuição da coordenação e do domínio do corpo, alteração da saúde. Se este quadro é certo em determinados casos, omite as diferenças individuais, a natureza e a amplitude dos câmbios físicos assim como a forma que estes se relacionam com fatores procedentes do ambiente e do meio social em geral.

Aragó (1995) refere que seria pouco correto acentuar unilateralmente o processo biológico no envelhecimento e coloca três razões principais: a) o ritmo do envelhecimento biológico é muito diverso segundo os indivíduos, pessoas com idade avançada gozam as vezes de melhor saúde que outras em plena idade madura ou inclusive jovens. Não se pode centrar nesta faceta biológica a noção de senectude; b) o declive biológico é real e em certa medida irreversível, mas não existe momento algum em que o crescimento psicológico do indivíduo deve cessar. O desenvolvimento psicológico pode conservar uma real independência com respeito a irreversibilidade relativa do processo biológico. O fator biológico terá maior ou menor peso conforme seja a personalidade do indivíduo; c) já em 1970, De Vries advertia contra o perigo

Estud. interdiscip. envelhec., Porto Alegre, v.2, p.35-47, 1999. 
de uma interpretação simplista e unidirecional a julgar a existência de uma mútua influência entre fatores fisiológicos e comportamentais.

Woodruff (1980) demonstrou a existência de uma mútua influência entre fatores comportamentais e fisiológicos como efeito da intervenção; segundo a autora, "( . . . ) se faz necessário modificar nossos possíveis conhecimentos acerca da plasticidade do organismo envelhecido tanto a nível fisiológico como conductual."

\subsection{Idade Psicológica}

Aragó (1995), fala da idade psicológica como função das modificações cognitivas e afetivas que produzem-se ao longo do transcurso temporal. Baltes; Willis (1982) insistem na “( . . ) existência de grande potencial latente para o rendimento intelectual na velhice", potencial latente que nunca chegaria a tornarse efetivo, mas que pode ser aproveitado sistematicamente mediante uma intervenção adequada (Cubas, 1984).

Para estudar as diferenças psicológicas entre jovens e velhos Mishara; Riedel (1995), propõem que cabe examinar a maneira como as experiências de uma população de idade avançada diferem das de uma população jovem. Acrescentando, ainda, que é evidente que se possui mais experiência aos 70 do que aos 40 anos. Mas a instrução se eleva, de tal sorte que certos sujeitos jovens podem ter mais experiência que os mais velhos.

No processo de envelhecimento a avaliação do futuro modifica-se, já que as expectativas de vida são curtas e o futuro adquire um peso que antes não tinha, o que repercute na organização da conduta. Segundo Neugarten; Havighurst; Tobin (1968), a partir de um determinado momento, “ ( . . . ) a vida se reorganiza em função do tempo que ainda tem por viver mais que do tempo transcorrido desde o nascimento." (p.97). Os câmbios psicológicos podem dividir-se em dois grupos: 1- os cognitivos, ou seja, aqueles que afetam a maneira de pensar assim como as capacidades e, 2 - os que concernem a afetividade e a personalidade. 


\section{Considerações Finais}

O clima político e social e a evolução geral do pensamento ao final do século XVIII e princípios do século XIX, marca o início do interesse pelo estudo de grupos sociais definidos em função de intervalos de idade. Surgiram os primeiros estudos científicos sobre o desenvolvimento da criança, do adolescente e do adulto. Estas origens são comuns, também, ao começo da psicologia científica. Todavia, frente ao grande desenvolvimento que experimentaram a psicologia da infância e da adolescência, a psicologia da idade adulta e da velhice permaneceram adormecidas até os anos sessenta e, inclusive setenta. Certamente, o final dos sessenta quando começa-se a reconhecer o envelhecimento como um campo científico, claramente definido.

Cabe colocar que a idade social, a biológica e a psicológica acentuam um aspecto, ou seja, cada uma tem suas vantagens e suas limitações, mas são complementarias. Assinalam as mútuas interrelações do biológico sobre o psicológico e social, e também, do psicológico e social sobre o biológico. Cada uma delas é a focalização de um aspecto da vida, o processo de envelhecimento com suas peculiaridades diferenciais e sociais e sua mútua superposição.

\section{Referências Bibliográficas}

1 AHMED, P. I.; KOLKER, A.; COELHO, G. V. Toward a New Definition of Health Overview. In: AHMED, P. I.; COELHO, G. V. (Eds.) Toward a New Definition of Health. Psichological Dimensions. New York, Plenum Press, 1979.

2 ANTONOVSKY, A. Unravelling the Mistery of Health. How People Manage Stress and Stay Well. San Francisco, Josset-Bass, 1987.

3 ARAGÓ, J. M. Aspectos psicosociales de la senectud. In: CARRETERO, M.; PALACIOS, J.; A. MARCHESI (Comp.). Psicología Evolutiva 3.

Adolescencia, madurez y senectud. Madrid, Alianza Editorial, 1995. 4 BALTES, P.B.; WILLIS, S. L. Plasticity and Enhancement of Intelectual Functioning in Old Age. In: CRAIK, F. I. M.; TREHUG, S.E. (Eds.). Aging and Cognitive Processes. New York, Plenum Press, 1982. 
5 BELLOCH, A. Conducta de salud y conducta de enfermedad. In: IBAÑEZ,

E.; BELLOCH, A. (Eds.). Psicología y Medicina. Valencia, Promolibro, 1989.

6 BIRREN, J. E. Handbook of Aging and the Individual. Chicago, University of Chicago Press, 1959.

7 BIRREN, J. E. Behavioral Theories of Aging. In: SHOCK, N. W. (Ed.). Aging-Some Social and Biological Aspects. Washington, D. C., American Assoc. Advac. Sci., 1960.

8 CARRETERO, M.; PALACIOS, J.; MARCHESI, A. Psicología evolutiva

3. Adolescencia, madurez y senectud. Madrid, Alianza, 1995.

9 COLL, C. El concepto de desarrollo en psicología evolutiva: aspectos epistemológicos. Infancia y Aprendizaje, Madrid, v.7, p.60-63, 1979.

10 COMFORT, A. The Biology of Senescence 3. ed. New York, Elsevier/ North Holland, 1979.

11 CUBAS, J. Memoria y envejecimiento: perspectivas contextuales como alternativa al modelo deficitario. Santiago de Compostela, 1984. Tesis Doctoral inédita.

12 De VRIES, H. A. Physiology of Exercise and Aging. In: WOODRUFF, D. S.; BIRREN, J. E. (Eds.). Aging: scientific perspectives and social issues. New York, Vand Nostrand-Reinhold, 1975.

13 ERIKSON, E. H. Identity and the Life Cycle. Psychological Issues, New York, N. Y., v.1, p.18-164, 1959.

14 HOGART, J. Glossary of Mental Health Care Terminology. Copenhague, Worl Health Organization, 1978.

15 JAHODA, M. Current Concepts of Positive Mental Health. New York, Basic Books, 1958. (Joit Commision of Mental Illiness and Health, Monograph Series, $\left.\mathrm{n}^{\circ} 1\right)$.

16 KART, C. S. The Reabilites of Aging: an introduction to Gerontology. Boston, Allyn and Bacon, 1990.

17 LEHR, U. Psicología de la senectud. Barcelona, Herder, 1980.

18 MASLOW, A. H. El hombre autorrealizado. Barcelona, Kairós, 1979.

19 MISHARA, B.L.; RIEDEL, R. G. El Proceso de envejecimiento. Madrid, Morata, 1995. 
20 NEUGARTEN, B. L.; HAVIGHURST, R. L.; TOBIN, S. S. Personality and Patterns of Aging. In: NEUGARTEN, B. L. (Eds.). Middle Age and Aging. Chicago, University of Chicago Press, 1968.

21 NEUGARTEN, B. L.; DATAN, N. Sociological Perspectives on the Life Cycle. In: BALTES, P.B.; SCHAIE, K. W. (Eds.). Life-span Developmental Psychology. Personality and Socialization. New York, Academic Press, 1973.

22 OREM, D. Nursing. Concepts of Pratice. New York, McGraw-Hill, 1980. 23 PARSONS, T. El sistema social. Madrid, Revista de Occidente, 1976.

24 PARSONS, T. Definitions of Health and Illness in the Light of American Values and Social Structure. In: CAPLAN, A. L.; ENGELHARDT, H. T.; McCARTHENY, J. J. (Eds.). Concepts of Health and Disease Interdisciplinary Perspectives. London, Addison-Wesley, 1981.

25 RODRÍGUEZ, S. La vejez: historia y actualidad. Salamanca, Ediciones Universidad de Salamanca, 1989.

26 SAN MARTÍN, H. Ecología humana y salud. El hombre y su ambiente. México, La Prensa Médica Mexicana, 1983.

27 TAYLOR, S. E. Seamos optimistas. Ilusiones positivas. Barcelona, Martínez Roca, 1991.

28 TRAUTNER, H. M. Lehrbuch der Entwicklungspsychologie. Göttingen, Hogrefe, 1979.

29 VEGA, J. L.; BUENO, B. Desarrollo adulto y envejecimiento. Madrid, Síntesis, 1996.

30 WARR, P. Work, Unemployment and Mental Health. Oxford, Clarence Press, 1987.

31 WOHLWILL, J. F. The Study of Behavioral Development. New York, Academic Press, 1973. 